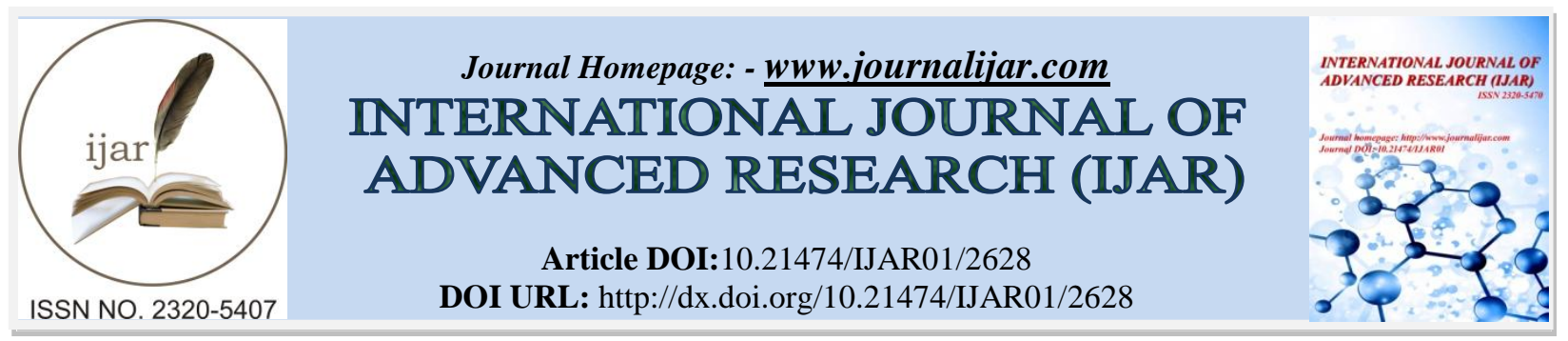

RESEARCH ARTICLE

\title{
SURGICAL MANAGEMENT OF HYPERPARATHYROIDISM; A SINGLE CENTER EXPERIENCE.
}

\author{
Abdullah A. AlArfaj MD ${ }^{1}$, Dalia A. Alarfaj MD ${ }^{2}$, Sayed I. ALI MD ${ }^{3}$ and Fahad A. Alsaab MD ${ }^{4}$ \\ 1,2\&4. Otolaryngology - Head and Neck surgery department. King Abdulaziz National Guard Hospital, King \\ Abdullah medical research center, AlAhssa, Saudi Arabia. \\ 3. Assistant Professor of BiostatisticsDepartment of Family Medicine \& community ,College Of Medicine King \\ Faisal University,AlAhssa , Saudi
}

\begin{abstract}
Arabia.
\end{abstract}
\section{Manuscript Info}

........................

Manuscript History

Received: 30 October 2016

Final Accepted: 29 November 2016

Published: December 2016

\section{Abstract}

Background: Hyperparathyroidism is over-activity of one or more of the parathyroid glands resulting in the excess production of parathyroid hormone. Primary hyperparathyroidism is the most common cause and has various clinical presentations, and a parathyroidectomy is considered to be the most effective treatment for this disorder.

Objective: To review the operative management of hyperparathyroidism from a single secondary hospital.

Method: This study hasa retrospective design whereby the charts of patients who underwent parathyroidectomies between 2010 and2015 were reviewed. We analysed the clinical manifestations, biochemical data, length of hospital stay, operative findings and post-operative complications.

Results: In total, the charts of 33 patients were examined, and the female to male ratio was determined to be 9: 2 and the mean age 55 years. The majority of patients $(51.5 \%)$ presented with bone pain and fatigue. Ultrasonography and a sestamibi parathyroid scan were used for pre-operative localisation in $81.8 \%$ of the patients, while the remainder underwentalternativemodality of localisation. Of the 33 patients who underwent surgery for parathyroid gland removal,78.8\% had a parathyroid adenoma. Finally, $75.8 \%$ of patients were free of any post-operative symptoms and the average length of hospital stay was 72 hours.

Conclusions: Primary hyperparathyroidism is a symptomatic disorder which commonly presents with skeletal and renal manifestations. The best surgical results are achieved through localisation of the parathyroid lesion.

Copy Right, IJAR, 2016,. All rights reserved.

\section{Introduction:-}

The four parathyroid glands are located within the neck, with two present on each side. These small glands secrete the hormone, parathyroid hormone (PTH), which helps the body to maintain a normal blood calcium level. When the blood calcium level drops, the normal response is for the parathyroid glands to release PTH in order to return the 
blood calcium level to normal. The blood calcium level is normally maintained by the kidneys, bones, and indirectly by the intestines. When the blood calcium level is increased, less PTH is released by the parathyroid glands, causing the kidneys to retain less calcium. These actions help maintain the body's calcium level within the normal range.

Primary hyperparathyroidism (PHP) is an endocrine disorder characterised by hypercalcemia and elevated, or inappropriate normal, levels of PTH. In early descriptions the disease was associated with the involvement of the kidneys (renal calculi and nephrocalcinosis), skeleton (osteitis fibrosa cystica, with pain, pathological fractures and deformity), and neuromuscular system (proximal neuromuscular weakness) (1). In addition, signs and symptoms affecting the psychiatric, cardiovascular, and gastrointestinal systems were frequent concomitants of the disease (2).

Today, PHPis usually an asymptomatic disorder, which is often diagnosed viaroutine biochemical screening of serum calcium levels (3).Generally, serum calcium levels are modestly elevated, often within $1 \mathrm{mg} / \mathrm{dl}$ of theupper limits of the normal range, and PTH concentrationsare1.5-2.0 times normal, while serum phosphorus levels are in the lower range of normal, and urinary calcium levels are at the upper limits of normal. If urinary calcium excretion is very low, familial hypocalciuric hypercalcemia should be ruled out before a diagnosis is made. Levels of 25hydroxy vitamin $\mathrm{D}$ tend to be atthe lower end of the normal range, whereas 1,25-dihydroxyvitamin D levels are elevated in $40 \%$ of patients(4).

Most patients with PHP are initially recognised when their serum calcium concentration is found to be elevated on a blood chemistry panel of tests. The 'intact' PTH level, as measured by animmuneradiometric assay (IRMA), is elevated or at levels that are inappropriate (in the upper range of normal), relative to the calcium level. Newer assays that measure full-length PTH protein (amino acids 1-84) mayhave greater sensitivity in the diagnosis of PHP than the older IRMA, in which large fragments of PTH, such as PTH(7-84), are also detected(5).To measure bone density, dual-energy X-ray absorptiometry is utilised. Patients with PHP tend to show a pattern of bone involvement that preferentially affects the cortical, as opposed to the cancellous skeleton (6).

Epidemiological studies have traditionally reported PHP incidence rate of 25-28 cases/100,000 population/year, and while the average incidence rate in white women aged over 60 years is approaching 190 cases/100,000/year(7). The results of a recent study have shown that the incidence rate of PHP is highest in blacks, followed by whites, then Asians, and lowest in Hispanics (8). In $80 \%$ of patients with PHP a benign, solitary adenoma is present, although in $2-5 \%$ of cases multiple adenomas may be found (9). In most of remaining patients a pathological process characterised by hyperplasia of all four parathyroid glands is involved, either sporadically or in association with multiple endocrine neoplasia type 1 or $2 \mathrm{a}$. .

The management of PHPis either via non-surgical or surgical treatment. Non-surgical treatment may be recommended for people who have no symptoms and whose blood calcium levels are only mildly elevated. In contrast, surgery is often recommended for people with symptoms or whose blood calcium levels are moderately elevated and there are signs of impaired kidney function or decreased bone density. Surgery is also recommended if a person is less than 50 years old or if periodic follow-up would be difficult. Traditional surgery is usually performed while the patients under anaesthesia and an incision is made in the lower neck measuring between 2.5 to $10 \mathrm{~cm}$ ( 1 to 5 inches). Usually, at least one abnormal-appearing gland is removed, while normal-appearing glands are left in situ.Other types of minimally invasive forms of surgery are performed in cases where only one abnormal parathyroid gland has been located via pre-operative imaging.

\section{Methodology:-}

This study has a retrospective design whereby the charts of 33 patients above the age of 20 years who underwent parathyroidectomies between 2010 and 2015 were reviewed. We analysed the clinical manifestations, biochemical and hormonal findings, length of hospital stay, operative findings, and post-operative complications. In addition, localisation techniques, histopathology and long-term disease status were also examined. Allstatistical analyses were performed using IBM statistics ver.21, and all results are expressed as the mean \pm the standard deviation (SD) and the median.

\section{Results:-}

Thirty three patients were analysed, consisting of 27 females and 6 males (ratio: 9: 2), who ranged in age from 23 to 85 years old (mean age 55 , median age 55). 
The major presenting symptoms in 17 (51.5\%) patients werebone pain and fatigue, whilehigh calcium levelswereobserved in $3(9.1 \%)$ patients, recurrent bone fractures in 1 (3\%) patient, renal calculi and neck swelling in $1(3 \%)$ patient, renal failure and a thyroid nodule in $1(3 \%)$ patient, recurrent renal stones in $2(6.1 \%)$ patients, a disc prolapse and bone pain in $1(3 \%)$ patient, lethargy in $2(6 \%)$ patients, multinodular goitre and dyspnea in $1(3 \%)$ patient, a palpable thyroid mass in $1(3 \%)$ patient, muscle pain in $1(3 \%)$ patient, and finally $2(3 \%)$ patients were diagnosed incidentally from their laboratory results(Figure 1). None of the patients had psychiatric symptoms or obvious neurological deficits, and gastrointestinal symptoms of nausea, vomiting and constipation were absent. None of the patients reported hypertension or hadhistory of either pancreatitis or palpable osteitis fibrosa cystica.

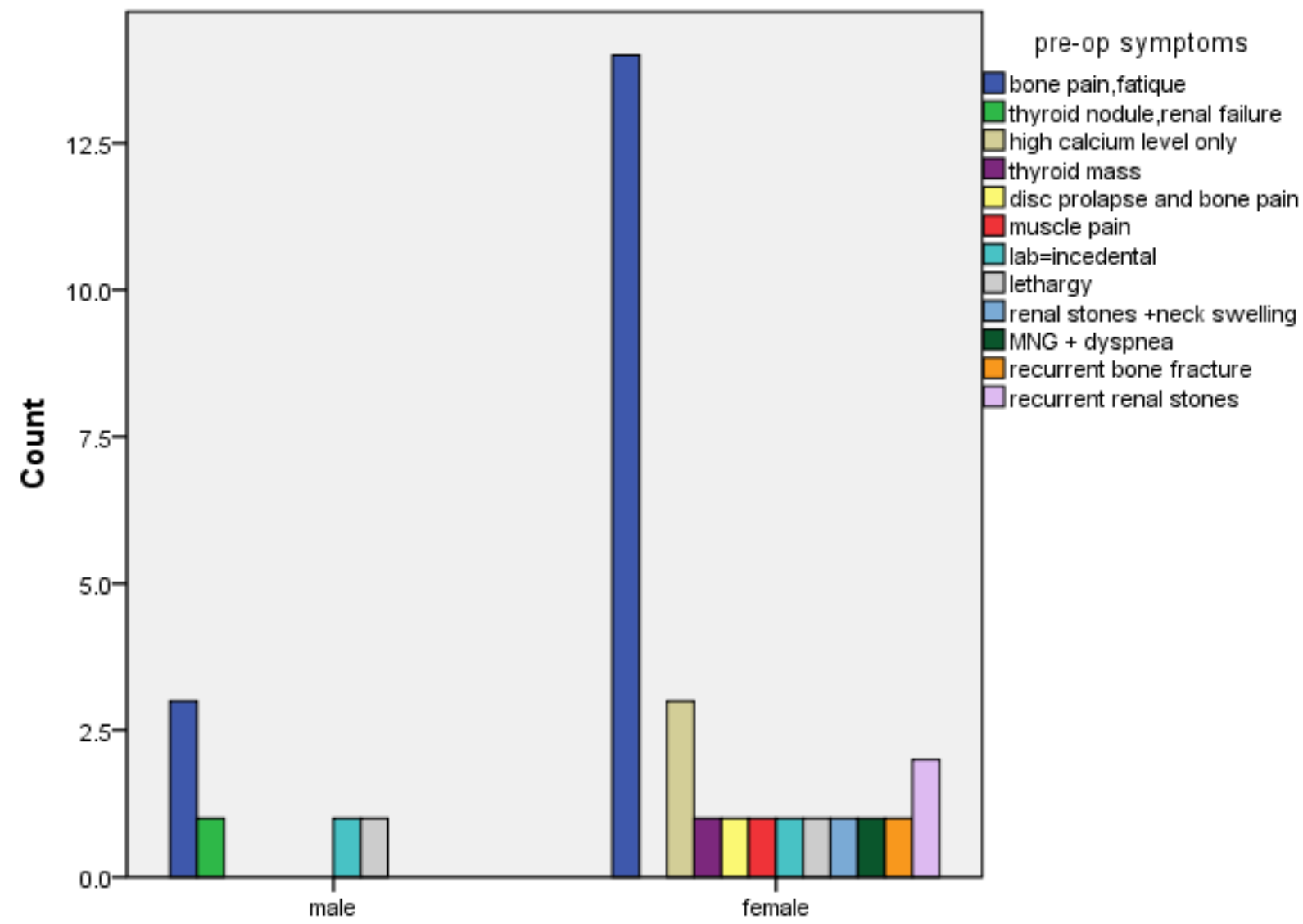

Figure 1:-Presenting symptoms

Serum biochemistry results showed elevated total corrected calcium levels (mean \pm SD: $2.92 \pm 0.33 \mathrm{mmol} / \mathrm{L}$ ), with lowered inorganic phosphorus levels (mean \pm SD: $0.93 \pm 0.19 \mathrm{mmol} / \mathrm{L}$ ). Levels of PTH were found to be elevated (mean \pm SD: 45.09 \pm 49.36 ; median 114.7, range: 1-228.4 pg/ml) (Table 1).

Table 1:- Biochemical and histological findings

\begin{tabular}{|l|l|l|}
\hline Variable & Mean (SD) & Median \\
\hline Corrected serum total calcium $(\mathrm{mmol} / \mathrm{l})$ & $2.92(0.33)$ & 2.82 \\
\hline Inorganic phosphorus & $0.93(0.19)$ & 0.91 \\
\hline parathyroid hormone & $45.09(49.36)$ & 27.2 \\
\hline Hyperplasia present & 7 & - \\
\hline Adenoma present & 26 & - \\
\hline
\end{tabular}

Pre-operative localisation was performed using ultrasonography (USG) and asestamibi parathyroid scan. In total, 27 $(81.8 \%)$ patients underwent pre-operative localisation, although $2(6.1 \%)$ patients only had USGand 3 (9.1\%) patients only had a sestamibi parathyroid scan.

A neck ultrasound revealed parathyroid hyperplasia and enlargement in 5 (15.2\%)patients, a left posterior inferior parathyroid adenoma in $4(12.2 \%)$ patients, a parathyroid nodule in $5(15.2 \%)$ patients, a right thyroid nodule or right 
adenoma in $1(3 \%)$ patient, a right lower increase in vascularity in $1(3 \%)$ patient, a hypoechoic area close to the right inferior pole in $1(3 \%)$ patient, a right upper hyperactive gland in $1(3 \%)$ patient, a prominent left inferior pole of the parathyroid gland in 1 (3\%)patient, and an undefined lesion was observed in3 (9.1\%)patients (Figure 2).

Skeletal involvement was studied using a B scan which revealed osteopenia in $11(9.1 \%)$ patients, osteoporosis in 4 (6\%) patients, sacroiliitis in $1(3 \%)$ patient, and an osteoarthritic joint in $1(3 \%)$ patient, while 6 patients (18.2) had both osteoporosis and osteopenia. In total,5 (15.2\%)patients had normal scans and 5 (15.2\%) patients underwent surgery without being subjected to a scan.

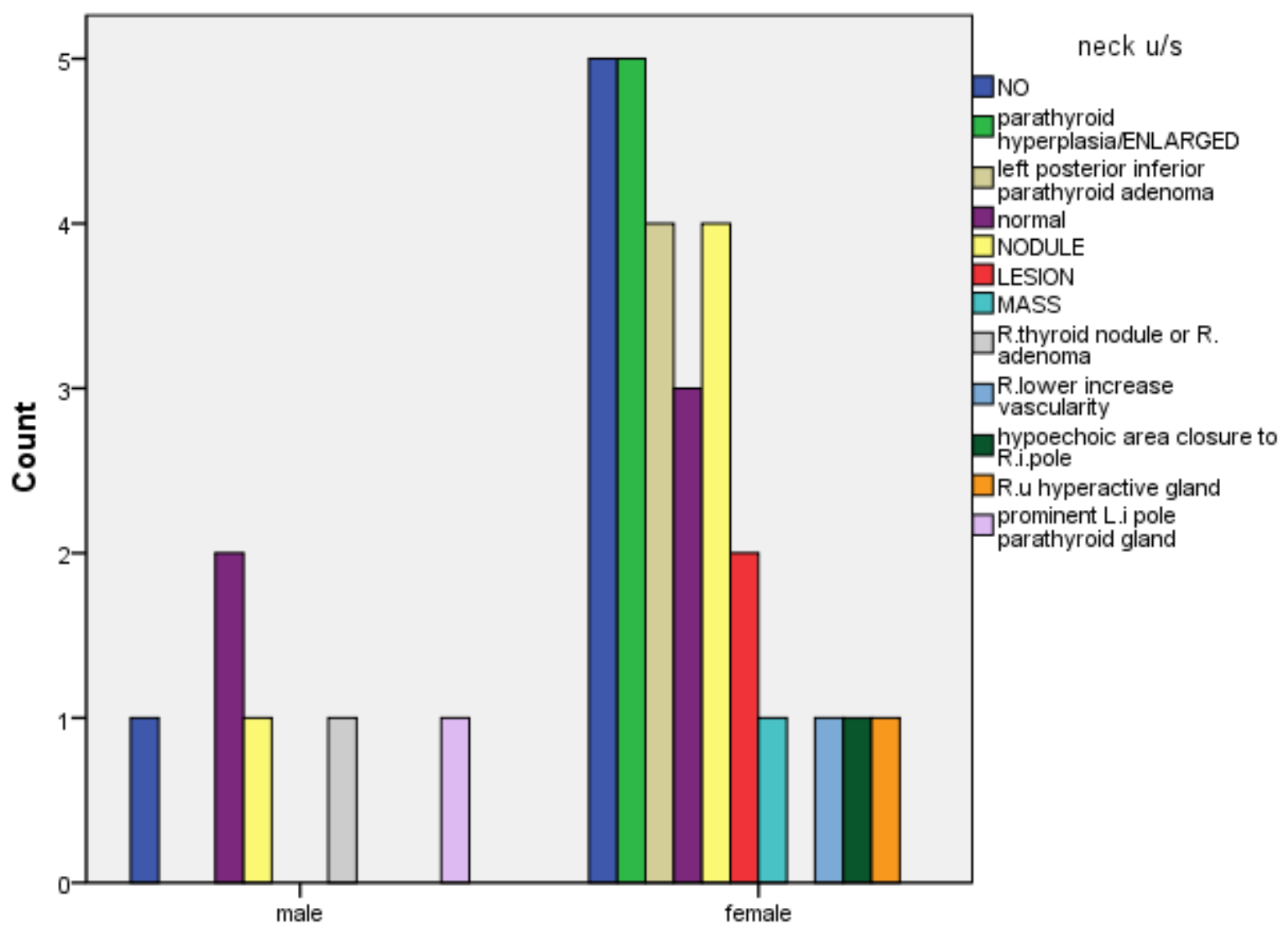

Figure 2:- Neck ultrasound findings.

Of the 33 patients who underwent surgery, 27(81.8\%) had a parathyroidectomy only,while6 (18.2) underwent a total thyroparathyroidectomy. Specimens were sent to for histopathology analysis, which confirmed that $7(21.2 \%)$ patients had parathyroid hyperplasia and 26(78.8\%) patients had parathyroid adenoma.

In terms of post-operative symptoms, 25 (75.8\%) patients experienced none, while $2(6.1 \%)$ patients had numbness in their face and upper limbs, 1 (3\%) patient had tingling in both hands, 1 (3\%) patient had perioral numbness , 1 (3\%) patient had mild neck pain, 1 (3\%) patient had hypercalcemia,1 (3\%)patienthad hypocalcaemia and 1 (3\%) patient complained of shortness of breath (Figure 3). 


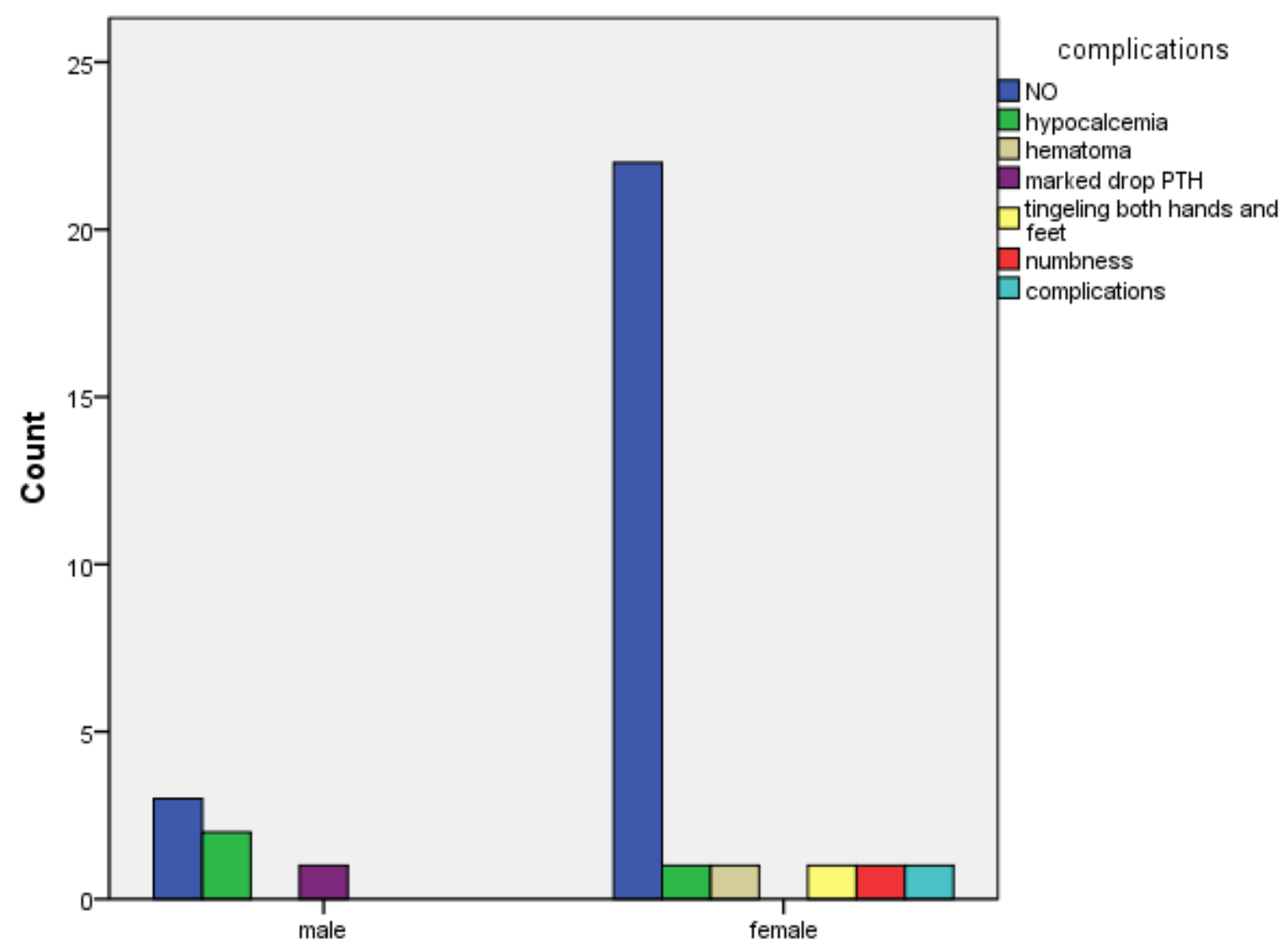

Figure 3:- Post-operative symptoms.

\section{Discussion:-}

As anticipated, PHP was more common in females than male, as showing in our study where there is a female to male ratio of 9: 2 and the mean age is55; these data are similar to North American and European data (10). In our study, nonspecific complaints, such as weakness, easily fatigued, and bone pain, were the most frequent presentations, followed by asymptomatic patients with incidental findings of hypercalcemia. Compared to other studies conducted in the US, the most common clinical presentation of PHP was asymptomatic PHP. The classical manifestations of PHP ('bones, stones, abdominal moans, and psychic groans') are unusualwithinWestern data and not present in our study (11).Recent studies indicate a reduced incidence of renal stones in PHP, and in our study this was found in less than $9 \%$ of patients. This is probablydue to routine calcium check-ups and the consequent early detection of asymptomatic cases of PHP(12).However, most patients with PHPtodayhave mild, non-specific symptoms, and the identification of their hypercalcemia is usually by chance (11).

Corrected mean serum calcium levels were slightly elevated $(2.92 \pm 0.33 \mathrm{mmol} / \mathrm{L})$, while mean serum inorganic phosphorus levels were to some extent lower than expected $(0.93 \pm 0.19 \mathrm{mmol} / \mathrm{L})$. This finding is in agreement with recent Western data, where in most asymptomatic patients the mean serum calcium concentration is less than 1.0 $\mathrm{mg} / \mathrm{dL}(0.25 \mathrm{mmol} / \mathrm{L})$, which is above the upper limit of the normal range and phosphate levels are not particularly low(13). As ina previously reported study, levels of PTH were markedly elevated (mean \pm SD: $45.09 \pm 49.36 \mathrm{pg} / \mathrm{ml}$ ) (14).

A four gland parathyroid exploration has traditionally been considered the gold standard for patients undergoing surgery for PHP. However, a more dedicated, minimally invasive approach to parathyroid surgery is being implemented within many centres today.Pre-operative parathyroid localisation studies are valuablein identifying the affectedparathyroid gland and minimise the surgical approach required. Localisation of an abnormal gland hassignificantly improved over the last decade, and numerous invasive and non-invasive procedures now exist. Preoperative localisation via USG and a sestamibi parathyroid scan was usedforall patients in this study. 
Surgery is considered to be the optimal curative treatment for patients with PHP, and post-operative follow-up of at least one year was available for all33 patients. Three patients underwent further surgery due to a recurrence of their symptoms; one had parathyroid gland hyperplasia, while the other two patients had a recurrent adenoma. In this study, the majority of parathyroidgland adenomas originated from the right inferior gland, and a previous study reporteda similar finding of adenomas predominantly arising from inferior glands. Post-operatively, our patients did not encounter any complications except for transient hypoparathyroidism, with hypocalcaemia symptoms observed in about $11 \%$ of our patients.

In conclusion, the presentation of PHP in our study occurred predominately in females in their fifties who had nonspecific symptoms (weakness, easily fatigued and bone pain) butpresented with classical biochemical abnormalities (hypercalcemia, hypophosphatemia and inappropriately elevated levels of PTH). Pre-operative localisation is very important in order to enable precise surgery with high successrates and fewer complications.

\section{References:-}

1. Mallette, L.E., et al. "Primary hyperparathyroidism: clinical and biochemical features."Medicine53.2 (1974): 127-146.

2. Silverberg, S.J., Walker, M.D. and Bilezikian, J.P. "Asymptomatic primary hyperparathyroidism."Journal of Clinical Densitometry16.1 (2013): 14-21.

3. Raue, F,.et al. "The role of the extracellullar calcium-sensing receptor in health and disease."Experimental and Clinical Endocrinology \&Diabetes 114.08 (2006): 397-405.

4. Silverberg, S.J.,etal."The effects of vitamin D insufficiency in patients with primaryhyperparathyroidism."American Journal of Medicine107(1999):561-567.

5. Silverberg, S.J., et al. "Clinical utility of an immunoradiometric assay for parathyroid hormone (1-84) in primary hyperparathyroidism.” Journal of Clinical Endocrinology \& Metabolism88.10 (2003): 4725-4730.

6. Silverberg, S.J., et al. "Skeletal disease in primary hyperparathyroidism." Journal of Bone and Mineral Research4.3 (1989): 283-291.

7. HeathIII, H. "Primary hyperparathyroidism: recent advances in pathogenesis, diagnosis, and management."Advances in Internal Medicine37 (1992): 275-293.

8. Yeh, M.W., et al. "Incidence and prevalence of primary hyperparathyroidism in a racially mixed population."Journal of Clinical Endocrinology \& Metabolism98.3 (2013): 1122-1129.

9. Part-Shane, E. (2001) "Parathyroid carcinoma." In The Parathyroids, $2^{\text {nd }}$ edn , 515-526 (EdsBilezikian JP et al.)New York: Academic Press

10. Yeh, M.W., et al. "Incidence and prevalence of primary hyperparathyroidism in a racially mixed population." Journal of Clinical Endocrinology \& Metabolism98.3 (2013): 1122-1129.

11. Silverberg, S.J. and Bilezikian, J.P."Evaluation and management of primary hyperparathyroidism."The Journal of Clinical Endocrinology \& Metabolism81.6 (1996): 2036-2040.

12. Heath, H. "Clinical spectrum of primary hyperparathyroidism: evolution with changes in medical practice and technology." Journal of Bone and Mineral Research6.S2 (1991): S63-S70.

13. Bilezikian, JP. and Silverberg, S.J."Asymptomatic primary hyperparathyroidism."New England Journal of Medicine350.17 (2004): 1746-1751.

14. Harinarayan, C. V., Gupta, N. and Kochupillai,N. "Vitamin D status in primary hyperparathyroidism in India."Clinical endocrinology43.3 (1995): 351-358. 